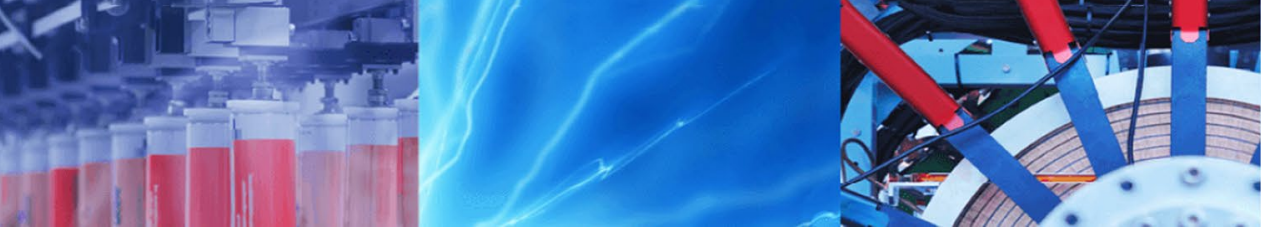

Research Article

\title{
Impact of Lorentz force on free convection flow of a viscous fluid past an infinite vertical plate
}

\author{
T. Sravan Kumar ${ }^{1}$ \\ (c) Springer Nature Switzerland AG 2019
}

\begin{abstract}
In this paper, an investigation carried out to study the effects of thermal diffusion and hall current, radiation on unsteady MHD free convective viscous, incompressible, electrically conducting chemically reacting fluid past an impulsively moving infinite vertical plate embedded in a fluid saturated porous medium with variable temperature and also with variable concentration under the influence of uniform transfers applied magnetic field. The dimensionless governing partial differential equations are tackled with the usual integral transform technique without any restriction. The results illustrated graphically for the velocity, temperature and concentration distributions based on the numerical computations are performed and also discussed the effects of various physical flow parameters on the skin-friction coefficient, the rate at which heat transfer and mass transfer changes near the surface in the fluid.
\end{abstract}

Keywords Magnetic field · Free convection · Hall current · Soret effect and Radiation

\section{Introduction}

The study of free convection flow during vertical surfaces embedded in a fluid-saturated porous medium is motivated by its practical applications in Astrophysics, Geophysics, and engineering. Among the applications of electrical power generation, thermal insulators, centrifugation filtration processes, ion propulsion, extraction of crude oil, heat exchanger devices, storage of nuclear wastes, catalytic chemical reactors, cooling of molten metal, and geothermal reservoirs. The mention is made of the research studies (Cramer and Pai [1], Cheng and Minkowycz [2], Nakayama and Koyama [3] Lai and Kulacki [4]). Hsieh et al. [5] have obtained an analytical solution for mixed convection from a vertical flat plate in saturated porous media. Nield and Kuznetsov [6] and Gorla and Chamkha [7] have examined the influence of nanoparticles on the natural convection boundary layer flow in a porous medium past a vertical plate. The topic of thermal convection in porous media has been reviewed through the articles by (Nield and Bejan [8] and Vafai [9], Pop and Ingham [10]).
In the present scenario, the problems on viscous incompressible electrically conducting with rotating fluid through porous medium have more physical significant attracted by many research. Among the applications of rotating flows in porous media is in the design of multi-pore fixer in a gas solid-fluid bed. A multi-pore fixer is a device, which is built from foramina's materials, compressed fibers, wire compacts, filter cloth, and sintered metal. Raptis and Kafouaias [11] have been studied heat transfer in flow through a porous medium bounded by an infinite vertical plate under the action of a magnetic field. Unsteady MHD natural convective viscous, incompressible, electrically conducting fluid through a porous medium bounded by an infinite vertical plate investigated Raptis [12]. Free convection flow along with an inclined plate embedded in a thermally stratified porous medium in the presence of a variable magnetic field by using finite difference method has been studied Chamkha [13]. Aldoss et al. [14], have obtained the strength of the magnetic parameter is found the effect on the shear stress on the plate in the case of hydromagnetic mixed convection flow over a vertical

T. Sravan Kumar, thavadasravankumar@gmail.com | 'Department of Mathematics, SAS, REVA University, Bangalore 560 064, India.

SN Applied Sciences (2019) 1:1257| https://doi.org/10.1007/s42452-019-1292-8 
plate embedded in a fluid saturated porous medium with the uniform magnetic field. Kim [15] have presented on unsteady magneto-hydrodynamic free convective heat transfer past a vertical plate through a porous medium in the presence of variable suction. Ibrahim et al. [16] considered unsteady, viscous, incompressible micropolar fluid past a moving vertical plate through a porous medium; the dimensionless equations were solved by a regular perturbation method. Alam and Rahman [17] examined the combined effects of thermal diffusion and diffusion thermo on MHD natural convective heat and mass transfer flow past a vertical porous plate in porous media by using the numerical technique. Makinde and Ogulu [18] have studied hydromagnetic mixed convection flow and mass transfer past a vertical plate embedded in the presence of a uniform transfer magnetic field. Makinde [19] investigated hydromagnetic boundary layer flow, heat and mass transfer past a vertical plate through a porous medium with constant heat flux by using the numerical technique.

The combined study, the effects of heat and mass transfer problems with radiation, chemical reaction are of importance in many processes, such as thermo-nuclear fusion, drying, cosmic flights, evaporation at the surface of a water body, propulsion systems, spacecraft, glass production, where the heat and mass transfer occurs simultaneously. Cess [20] has discussed the interaction of radiation with natural convection heat transfer. Hossain and Takhar [21] examined the effects of radiation on the free and forced convection along a vertical plate with uniform heat transfer at the surface by using the numerical technique. Thermal radiation effects on the free and forced convection from a horizontal flat plate in a porous medium. Both a cold surfacing facing downward and a hot surfacing upward are analyzed Bakier and Gorla [22]. Thermal radiation and buoyancy effects on hydromagnetic flow over an accelerating permeable surface with heat source or absorption have been studied Chamkha [23]. The combined effects of thermal radiation and viscous dissipation on unsteady MHD free convection past a vertical plate in a porous medium with time-dependent suction has been investigated Israel-Cookey et al. [24]. Muthucumaraswamy [25] has obtained an analytical solution for the effects of chemical reaction on impulsively moving vertical plate with variable mass diffusion. Makinde and Sibanda [26] investigated the effects of radiation and mass transfer flow of variable viscosity fluid past a vertical embedded porous plate with uniform transform magnetic field. Mostafa and Mahmoud [27] have studied thermal radiation effect on hydromagnetic natural convection flow past a vertical plate with temperature dependent viscosity. Rushi Kumar et al. [28] investigated the exact solution of Soret and radiation effects on unsteady natural convection flow in the presence of magnetic field. Nandkeolyar et al. [29] studied combine the effects of heat and mass transfer flow past an infinite vertical plate through a porous medium with ramped wall temperature by using the Laplace transform technique. Seth et al. [30] have been studied hydromagnetic free convection flow heat and mass transfer past a moving vertical plate with a ramped wall temperature. Das et al. [31] investigated the effects of Hall current in hydromagnetic natural convection boundary layer flow past a vertical plate. Seth et al. [32] have obtained an analytical solution for the effects of Hall current and rotation on natural convection uniform temperature and mass transfer flow past a moving vertical plate. Seth et al. [33] and Takha et al. [34] has studied the effects of Hall current, radiation and rotation on unsteady hydromagnetic free convection flow with heat and mass transfer past a moving vertical plate in the presence of chemical reaction. Mythreye et al. [35] the effects of chemical reaction on unsteady hydromagnetic free convective heat and mass transfer past a vertical permeable moving plate with heat absorption by solving regular perturbation technique.

The study of magnetohydrodynamics with heat transfer and thermal radiation has gained great attention from researchers due to its various applications in materials processing, astrophysics and geophysics. Magneto-hydrodynamics involves the interactions of electrically conducting fluids and magnetic fields. Lately, many advanced devices that utilize the MHD interaction have been made, such as propulsion units, power generator, electron beam dynamics, travelling wave tubes, MHD pumps, and MHD bearing. Hence, it is essential to understand the MHD heat transfer of viscous fluids with radiation effect. Magneto-hydrodynamic convective flow issues are very imperative and some of the works on chemical engineering, planetary and stellar magnetospheres and aeronautics can be found in (Rashad et al. [36], Bhatti et al. [37], Yuan et al. [38-40]).

Flow in permeable media was studied through various examinations in the middle of past two decades. The findings of the investigated were showed that subject was strengthened to a large extent of thermally determined streams in permeable media. It has various applications in chemical and mechanical engineering, specifically in electrochemistry, soil matrix heat exchangers, microelectronics cooling, food processing and storage, non-nuclear waste, thermal insulations, fibrous insulation, geophysical systems, groundwater hydrology, the design of pebble bed nuclear reactors, geothermal systems, wall cooled catalytic reactors and energy-efficient drying process.

The aim of the present chapter is to study the effects of thermal diffusion, Hall current and radiation on unsteady MHD free convective viscous, incompressible, electrically conducting chemically reacting fluid past an impulsively moving infinite vertical plate embedded in a fluid saturated porous medium with variable temperature. It is also with variable concentration (how the mass is diffused from the plate) under the influence of uniform transverse 
magnetic field even though this situation involves in many engineering applications such as centrifugation filtration processes, nuclear power reactors, plasma physics, designing of electromagnetic device and cosmical flight. The solutions were determined to the derived dimensionless governing partial differential equations using integral transformation approach. Hence, plotted through displayed analytical results on the flow transport, temperature and concentration along with the rate of heat transfer, the rate of mass transfer and the skin-friction in terms of exponential and complementary error functions.

\section{Formulation of the problem}

In this paper, studies the effects of radiation, Hall current and thermal-diffusion (Soret effect) on unsteady MHD free convection of flow of a viscous incompressible, electrically, conducting, radiating fluid past an impulsively started infinite plate with variable temperature and mass diffusion in the presence of transverse applied a magnetic field (Fig. 1). The $x^{\prime}$-axis is taken along the plate in vertically upward direction and the $y^{\prime}$-axis is taken normal to it the $z^{\prime}$-axis perpendicular to the $x^{\prime} y^{\prime}$-plane. Both fluid and plate are in rigid body rotation with an angular velocity $\Omega$ about the $z^{\prime}$ -axis. Initially, it is assumed that the plate and surrounding fluid are at the same temperature and concentration in a stationary condition for all the points in entire flow region $y^{\prime} \geq 0$. At the time $t^{\prime}>0$, the plate is given an impulsive motion with velocity $u^{\prime}=u_{0}$ in its own plane. At the same time, the plate temperature is raised linearly with time $t$ and also the mass is diffused from the plate linearly with time $t$. A magnetic field of uniform strength $B_{0}$ is assumed to be applied normal to the direction of the flow the induced magnetic field is assumed to be neglected as the magnetic Reynolds number of the flow is taken to be very small. For free convection flow, it is also assumed that in the energy equation the dissipation of viscosity is ignored. It is viewed as the density difference and species concentration effects in the body forces term consequently in Boussinesq's approximation. The fluid is assumed to be gray emitting and absorbing electromagnetic waves but not-scattering medium.

Under the above assumption, the governing boundary layer equations are given by

Under the above assumptions the equations governing the flow field are given by

$$
\begin{aligned}
\frac{\partial u^{\prime}}{\partial t^{\prime}}+2 \Omega w^{\prime}= & v \frac{\partial^{2} u^{\prime}}{\partial y^{\prime 2}}-\frac{\sigma B_{0}^{2}}{\rho\left(1+m^{2}\right)}\left(u^{\prime}+m w^{\prime}\right) \\
& +g \beta^{\prime}\left(T^{\prime}-T_{\infty}^{\prime}\right)+g \beta^{*}\left(C^{\prime}-C_{\infty}^{\prime}\right)-\frac{v u^{\prime}}{K^{\prime}}
\end{aligned}
$$

$\frac{\partial w^{\prime}}{\partial t^{\prime}}-2 \Omega u^{\prime}=v \frac{\partial^{2} w^{\prime}}{\partial y^{\prime 2}}+\frac{\sigma B_{0}^{2}}{\rho\left(1+m^{2}\right)}\left(-w^{\prime}+m u^{\prime}\right)-\frac{v w^{\prime}}{K^{\prime}}$

$\frac{\partial T^{\prime}}{\partial t^{\prime}}=\frac{1}{\rho c_{p}}\left(k \frac{\partial^{2} T^{\prime}}{\partial y^{\prime 2}}-\frac{\partial q_{r}^{\prime}}{\partial y^{\prime}}\right)$

$\frac{\partial C^{\prime}}{\partial t^{\prime}}=D \frac{\partial^{2} C^{\prime}}{\partial y^{\prime 2}}+D_{1} \frac{\partial^{2} T^{\prime}}{\partial y^{\prime 2}}-K_{r}^{\prime}\left(C^{\prime}-C_{\infty}^{\prime}\right)$

The following initial and boundary conditions are as follows

Fig. 1 Physical model and coordinate system

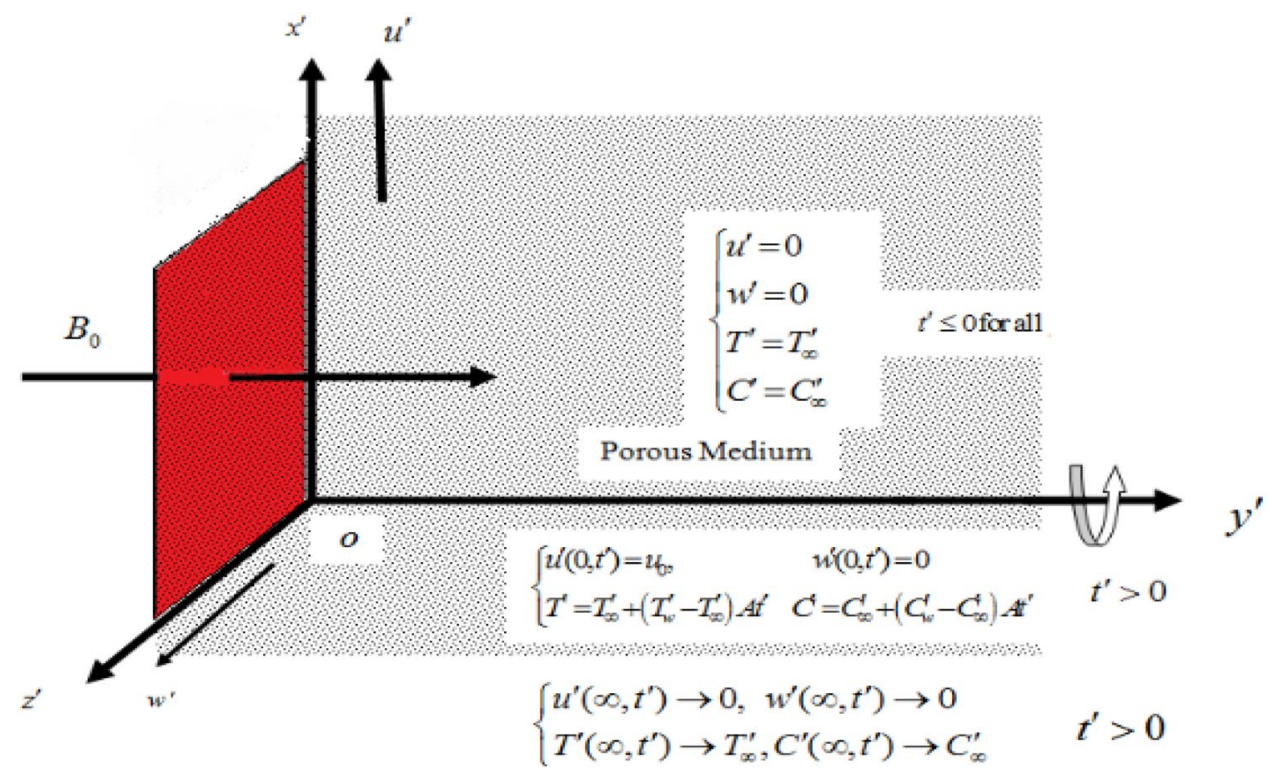


$t^{\prime} \leq 0: u^{\prime}=0, \quad w^{\prime}=0 \quad T^{\prime}=T_{\infty^{\prime}}^{\prime} \quad C^{\prime}=C_{\infty^{\prime}}^{\prime} \quad$ for all $y^{\prime}$

$t^{\prime}>0: u^{\prime}=u_{0}, \quad w^{\prime}=0, \quad T^{\prime}=T_{\infty}^{\prime}+\left(T_{w}^{\prime}-T_{\infty}^{\prime}\right) A t^{\prime}$,

$C^{\prime}=C_{\infty}^{\prime}+\left(C_{w}^{\prime}-C_{\infty}^{\prime}\right) A t^{\prime}$ at $y^{\prime}=0$

$u^{\prime} \rightarrow 0, \quad w^{\prime} \rightarrow 0, \quad T^{\prime} \rightarrow T_{\infty^{\prime}}^{\prime} \quad C^{\prime} \rightarrow C_{\infty^{\prime}}^{\prime} \quad$ as $y^{\prime} \rightarrow \infty$

where $A=\frac{u_{0}^{2}}{v}$ the local radiant for the case of an optically thin gray gas is expressed by

$\frac{\partial q_{r}}{\partial y^{\prime}}=-4 a^{*} \sigma\left(T_{\infty}^{\prime 4}-T^{\prime 4}\right)$

It is assumed that the surface conditions within the flow are sufficiently small and that $T^{\prime 4}$ may be expressed as a linear function of the surface conditions. This is obtained by expanding $T^{\prime 4}$ in a Taylor series about $T_{\infty}^{\prime}$ and ignore the higher order terms, we get

$$
\begin{aligned}
& T^{4} \cong T_{\infty}^{4}+4 T_{\infty}^{3}\left(T-T_{\infty}\right) \\
& T^{4} \cong T_{\infty}^{4}+4 T_{\infty}^{3}-4 T_{\infty}^{4} \\
& T^{\prime 4} \cong 4 T_{\infty}^{\prime 3} T^{\prime}-3 T_{\infty}^{\prime 4}
\end{aligned}
$$

The above Eqs. (6) and (7), Eq. (3) reduces to

$\rho c_{p} \frac{\partial T^{\prime}}{\partial t^{\prime}}=k \frac{\partial^{2} T^{\prime}}{\partial t^{\prime 2}}+16 a^{*} \sigma T_{\infty}^{\prime 3}\left(T_{\infty}^{\prime}-T^{\prime}\right)+\frac{D_{m} K_{T} \rho}{C_{s}} \frac{\partial^{2} C^{\prime}}{\partial y^{\prime 2}}$

The following non-dimensional quantities are introduced

$$
\begin{aligned}
u & =\frac{u^{\prime}}{u_{0}}, \quad w=\frac{w^{\prime}}{u_{0}}, \quad t=\frac{t^{\prime} u_{0}^{2}}{v}, \quad y=\frac{y^{\prime} u_{0}}{v}, \quad \theta=\frac{T^{\prime}-T_{\infty}^{\prime}}{T_{w}^{\prime}-T_{\infty}^{\prime},} \\
C & =\frac{C^{\prime}-C_{\infty}^{\prime}}{C_{w}^{\prime}-C_{\infty}^{\prime}}, \quad K^{2}=\Omega v / u_{0}^{2} \quad G r=\frac{g \beta v\left(T_{w}^{\prime}-T_{\infty}^{\prime}\right)}{u_{0}^{3}}, \\
G m & =\frac{g \beta^{*} v\left(C_{w}^{\prime}-C_{\infty}^{\prime}\right)}{u_{0}^{3}}, \quad \operatorname{Pr}=\frac{\rho v C_{\rho}}{k}, \quad S o=\frac{D_{1}\left(T_{w}^{\prime}-T_{\infty}^{\prime}\right)}{v\left(C_{w}^{\prime}-C_{\infty}^{\prime}\right)}, \\
S C & =\frac{v}{D^{\prime}} \quad M=\frac{\sigma B_{0}^{2} v}{\rho u_{0}^{2}}, \quad R=\frac{16 a^{*} v^{2} \sigma T_{\infty}^{\prime 3}}{k u_{0}^{2}}, \quad K=\frac{K^{\prime} u_{0}^{2}}{v^{2}}, \quad K_{r}=\frac{K_{r}^{\prime} v}{u_{0}^{2}}
\end{aligned}
$$

$\frac{\partial w}{\partial t}-2 K^{2} u=\frac{\partial^{2} w}{\partial y^{2}}+\frac{M(m u-w)}{\left(1+m^{2}\right)}-\frac{w}{K}$

$\frac{\partial \theta}{\partial t}=\frac{1}{\operatorname{Pr}}\left(\frac{\partial^{2} \theta}{\partial y^{2}}-R \theta\right)$

$\frac{\partial C}{\partial t}=\frac{1}{S c} \frac{\partial^{2} C}{\partial y^{2}}+S o \frac{\partial^{2} \theta}{\partial y^{2}}-K_{r} C$

The initial and boundary conditions (5), in dimensionless form are as follows:

$t \leq 0: u=0, \quad w=0, \quad \theta=0, \quad C=0$ for all $y$

$t>0: u=1, \quad w=0, \quad \theta=t, \quad C=t \quad$ at $y=0$

$u \rightarrow 0, \quad w \rightarrow 0, \quad \theta \rightarrow 0, \quad C \rightarrow 0 \quad$ as $y \rightarrow \infty$

Combining Eqs. (10) and (11), we obtained

$\frac{\partial H}{\partial t}-2 i K^{2} H=\frac{\partial^{2} H}{\partial y^{2}}+G r \theta+G m C-F H-\frac{H}{K}$

where $H=u+i w$ and $F=\frac{M(1-i m)}{1+m^{2}}$.

The initial and boundary conditions (14), in compact form, are given by

$t \leq 0: H=0, \quad \theta=0, \quad C=0$ for all $y$

$t>0: H=1, \quad \theta=t, \quad C=t \quad$ at $y=0$

$H \rightarrow 0, \quad \theta \rightarrow 0, \quad C \rightarrow 0 \quad$ as $\quad y \rightarrow \infty$

Equations (12), (13) and (15), governed to the boundary conditions (16) are solved by usual integral transform technique and the solutions are expressed in terms of exponential and complementary error functions.

$\theta(y, t)=\left[\begin{array}{c}\left(\frac{t}{2}+\frac{y \operatorname{Pr}}{4 \sqrt{R}}\right) \exp (y \sqrt{R}) \operatorname{erfc}\left(\frac{y \sqrt{\mathrm{Pr}}}{2 \sqrt{t}}+\sqrt{\frac{R t}{\operatorname{Pr}}}\right) \\ +\left(\frac{t}{2}-\frac{y \operatorname{Pr}}{4 \sqrt{R}}\right) \exp (y-\sqrt{R}) \operatorname{erfc}\left(\frac{y \sqrt{\operatorname{Pr}}}{2 \sqrt{t}}-\sqrt{\frac{R t}{\operatorname{Pr}}}\right)\end{array}\right]$

Equations (1), (2), (4) and (8), in non-dimensional from, become

$\frac{\partial u}{\partial t}+2 K^{2} w=\frac{\partial^{2} u}{\partial y^{2}}-\frac{M(m w+u)}{\left(1+m^{2}\right)}-\frac{u}{K}+G r \theta+G m C$

$$
\begin{aligned}
C(y, t)= & \left(1-a_{5}\right) B_{1}+\frac{\left(a_{4}-a_{6}\right)}{2} B_{2}+\frac{\left(a_{6}-a_{4}\right) \mathrm{e}^{\left(-a_{3} t\right)}}{2} B_{3} \\
& +\frac{\left(a_{6}-a_{4}\right)}{2} B_{4}+a_{5} B_{5}+\frac{\left(a_{4}-a_{6}\right) \mathrm{e}^{\left(-a_{3} t\right)}}{2} B_{6}
\end{aligned}
$$

$$
\begin{aligned}
H(y, t)= & \frac{A_{0}}{2} B_{7}+A_{1} B_{8}+\frac{A_{2} \exp \left(-a_{8} t\right)}{2} B_{9}+\frac{A_{3} \exp \left(-a_{10} t\right)}{2} B_{10}+\frac{A_{4} \exp \left(-a_{3} t\right)}{2} B_{11}+\frac{A_{5}}{2} B_{12} \\
& +A_{6} B_{13}+\frac{A_{7} \exp \left(-a_{8} t\right)}{2} B_{14}+\frac{A_{8} \exp \left(-a_{10} t\right)}{2} B_{15} \frac{A_{9} \exp \left(-a_{3} t\right)}{2} B_{16}+\frac{A_{10}}{2} B_{17}+A_{11} B_{18} \\
& +\frac{A_{12} \exp \left(-a_{10} t\right)}{2} B_{19}+\frac{A_{13} \exp \left(-a_{3} t\right)}{2} B_{20}
\end{aligned}
$$


where

$$
\begin{aligned}
& a_{0}=\frac{-S C S O R}{\operatorname{Pr}-S c}, \quad a_{1}=\frac{-S C S O \operatorname{Pr}}{\operatorname{Pr}-S c}, \quad a_{3}=\frac{R-S c K_{r}}{\operatorname{Pr}-S c}, \quad a_{4}=\frac{a_{0}}{a_{3}^{2}}, \quad a_{5}=\frac{a_{0}}{a_{3}}, \quad a_{6}=\frac{a_{1}}{a_{3}}, \quad a_{7}=\frac{-G r}{\operatorname{Pr}-1}, \quad a_{8}=\frac{R-b}{\operatorname{Pr}-1}, \\
& a_{9}=\frac{-G m}{S c-1}, \quad a_{10}=\frac{K_{r} S c-b}{S c-1}, \quad a_{11}=\frac{a_{0} G m}{S c-1}, \quad a_{12}=\frac{G m a_{1}}{S c-1}, \quad a_{13}=\frac{-a_{0} G m}{\operatorname{Pr}-1}, \quad a_{14}=\frac{-a_{1} G m}{\operatorname{Pr}-1}, \quad a_{15}=\frac{a_{7}}{a_{8}^{2}}, \quad a_{16}=\frac{a_{7}}{a_{8}}, \\
& a_{17}=\frac{a_{9}}{a_{10}^{2}}, \quad a_{18}=\frac{a_{9}}{a_{10}}, \quad a_{19}=\frac{a_{11}\left(a_{10}+a_{3}\right)}{a_{3}^{2} a_{10}^{2}}, \quad a_{20}=\frac{a_{11}}{a_{3} a_{10}}, \quad a_{21}=\frac{a_{11}}{a_{3}^{2}\left(a_{3}-a_{10}\right)}, \quad a_{22}=\frac{a_{11}}{a_{10}^{2}\left(a_{3}-a_{10}\right)}, \quad a_{23}=\frac{a_{12}}{a_{3} a_{10}}, \\
& a_{24}=\frac{a_{12}}{a_{3}\left(a_{3}-a_{10}\right)}, \quad a_{25}=\frac{a_{12}}{a_{10}\left(a_{3}-a_{10}\right)}, \quad a_{26}=\frac{a_{13}\left(a_{3}+a_{8}\right)}{a_{3}^{2} a_{8}^{2}}, \quad a_{27}=\frac{a_{13}}{a_{3} a_{8}}, \quad a_{28}=\frac{a_{13}}{a_{3}^{2}\left(a_{3}-a_{8}\right)}, \quad a_{29}=\frac{a_{13}}{a_{8}^{2}\left(a_{3}-a_{8}\right)}, \\
& a_{30}=\frac{a_{14}}{a_{2} a_{8}}, \quad a_{31}=\frac{a_{14}}{a_{3}\left(a_{3}-a_{8}\right)}, \quad a_{32}=\frac{a_{14}}{a_{8}\left(a_{3}-a_{8}\right)}, \quad A_{0}=\left(1+a_{15}+a_{17}+a_{19}-a_{23}+a_{26}-a_{30}\right), \quad A_{1}=\left(-a_{16}-a_{18}-a_{20}-a_{27}\right), \\
& A_{2}=\left(-a_{15}+a_{32}\right), \quad A_{3}=\left(-a_{17}-a_{22}+a_{25}-a_{29}\right), \quad A_{4}=\left(a_{21}-a_{24}+a_{28}-a_{31}\right), \quad A_{5}=\left(-a_{15}-a_{26}+a_{30}\right), \\
& A_{6}=\left(a_{16}+a_{27}\right), \quad A_{7}=\left(a_{15}-a_{32}\right), \quad A_{8}=\left(a_{29}-a_{25}\right), \quad A_{9}=\left(-a_{28}+a_{31}\right), \quad b=N+\frac{1}{K}-2 i K^{2}, \\
& A_{10}=\left(-a_{17}-a_{19}+a_{23}\right), \quad A_{11}=\left(a_{18}+a_{20}\right), \quad A_{12}=\left(a_{17}+a_{22}\right), \quad A_{13}=\left(-a_{21}+a_{24}\right) .
\end{aligned}
$$

\subsection{Skin-friction}

From velocity field, the expression for skin-friction at the plate which is given in non-dimensional form as follows:

$$
C f=-\left[\frac{\partial H}{\partial y}\right]_{y=0}
$$

From Eqs. (19) and (20), we get the expression for skinfriction at the plate as follows:

$$
\begin{aligned}
& C f=A_{0}\left[-\exp (-b t) \frac{1}{\sqrt{\pi t}}-\sqrt{b} \operatorname{erf}(\sqrt{b t})\right]+A_{1}\left[-2 \exp (-b t) \sqrt{\frac{t}{\pi}}-2 t \sqrt{b} \operatorname{erf}(\sqrt{b t})+\frac{1}{\sqrt{b}} \operatorname{erf}(\sqrt{b t})\right] \\
& +A_{2} \exp \left(-a_{8} t\right)\left[\left(-\exp \left(-b t+a_{8} t\right)\right) \frac{1}{\sqrt{\pi t}}-\sqrt{b-a_{8}} \operatorname{erf}\left(\sqrt{b t-a_{8} t}\right)\right] \\
& +A_{3} \exp \left(-a_{10} t\right)\left[\left(-\exp \left(-b t+a_{10} t\right)\right) \frac{1}{\sqrt{\pi t}}-\sqrt{b-a_{10}} \operatorname{erf}\left(\sqrt{b t-a_{10} t}\right)\right] \\
& +A_{4} \exp \left(-a_{3} t\right)\left[\left(-\exp \left(-b t+a_{3} t\right)\right) \frac{1}{\sqrt{\pi t}}-\sqrt{b-a_{3}} \operatorname{erf}\left(\sqrt{b t-a_{3} t}\right)\right]
\end{aligned}
$$

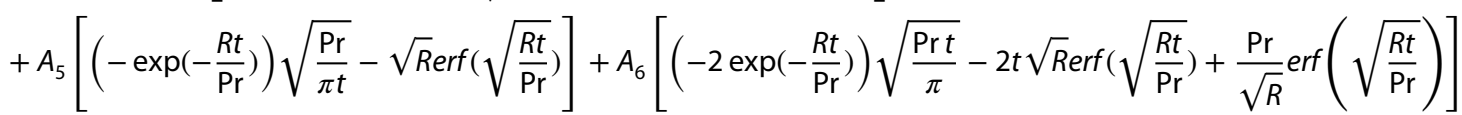

$$
\begin{aligned}
& +A_{7} \exp \left(-a_{8} t\right)\left[\left(-\exp \left(-\frac{R t}{\operatorname{Pr}}+a_{8} t\right)\right) \sqrt{\frac{\operatorname{Pr}}{\pi t}}-\sqrt{R-a_{8} \operatorname{Pr} e r f}\left(\sqrt{\frac{R t}{\operatorname{Pr}}-a_{8} t}\right)\right] \\
& +A_{9} \exp \left(-a_{3} t\right)\left[\left(-\exp \left(-\frac{R t}{\operatorname{Pr}}+a_{3} t\right)\right) \sqrt{\frac{\operatorname{Pr}}{\pi t}}-\sqrt{R-a_{3} \operatorname{Pr} e r f}\left(\sqrt{\frac{R t}{\operatorname{Pr}}-a_{3} t}\right)\right] \\
& +A_{10}\left[\left(-\exp \left(-K_{r} t\right)\right) \sqrt{\frac{S c}{\pi t}}-\sqrt{K_{r} S \operatorname{cerf}}\left(\sqrt{K_{r} t}\right)\right]+A_{11}\left[-2\left(-\exp \left(-K_{r} t\right)\right) \sqrt{\frac{S c t}{\pi}}-2 t \sqrt{K_{r} S \operatorname{erf}}\left(\sqrt{K_{r} t}\right)+\sqrt{\frac{S c}{K_{r}}} \operatorname{erf}\left(\sqrt{K_{r} t}\right)\right] \\
& +A_{12} \exp \left(-a_{10} t\right)\left[\left(-\exp \left(-K_{r} t+a_{10} t\right)\right) \sqrt{\frac{S c}{\pi t}}-\sqrt{K_{r} S c-a_{10} \operatorname{Sc} e r f}\left(\sqrt{K_{r} t-a_{10} t}\right)\right] \\
& +A_{13} \exp \left(-a_{3} t\right)\left[\left(-\exp \left(-K_{r} t+a_{3} t\right)\right) \sqrt{\frac{S c}{\pi t}}-\sqrt{K_{r} S c-a_{3} S c e r f}\left(\sqrt{K_{r} t-a_{3} t}\right)\right]
\end{aligned}
$$


From Eqs. (17) and (22), we get the rate of change of heat transfer at the plate as follows:

$N u=-\left[-t \sqrt{R} \operatorname{erf} \sqrt{\frac{R t}{\mathrm{Pr}}}-\sqrt{\frac{t \mathrm{Pr}}{\pi}} \exp \left(-\frac{R t}{\mathrm{Pr}}\right)+\frac{\mathrm{Pr}}{2 \sqrt{R}} \operatorname{erf} \sqrt{\frac{R t}{\mathrm{Pr}}}\right]$

\subsection{Sherwood number}

From concentration field, we study the rate of change of mass transfer at the plate which is given in non-dimensional form as follows:

$$
S h=-\left[\frac{\partial C}{\partial y}\right]_{y=0}
$$

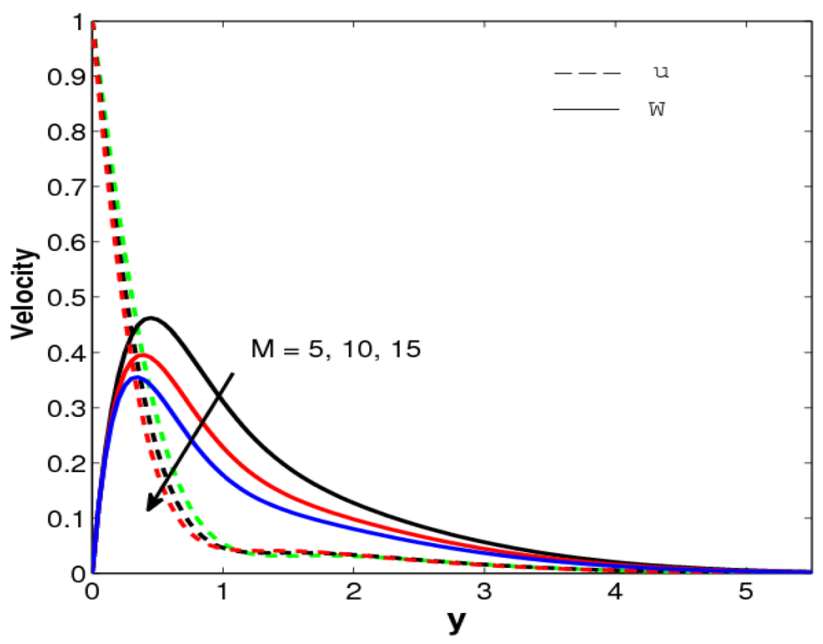

From Eqs. (18) and (24) we get the rate of change of mass transfer at the plate as follows:

Fig. 2 Transverse velocity profiles for different $M$

$$
\begin{aligned}
S h= & \left(1-a_{5}\right)\left[t \sqrt{K_{r} S c} \operatorname{erf} \sqrt{K_{r} t}+\sqrt{\frac{t S c}{\pi}} \exp \left(-K_{r} t\right)+\frac{\sqrt{S c}}{2 \sqrt{K_{r}}} \operatorname{erf} \sqrt{K_{r} t}\right] \\
& +\left(a_{4}-a_{6}\right)\left[\sqrt{\frac{S c}{\pi t}} \exp \left(-K_{r} t\right)+\sqrt{K_{r} S c} \operatorname{erf} \sqrt{K_{r} t}\right] \\
& +\left(-a_{4}+a_{6}\right) \exp \left(-a_{3} t\right)\left[\sqrt{\frac{S c}{\pi t}} \exp \left(k-a_{3}\right) t+\sqrt{\left(K_{r}-a_{3}\right) S c} \operatorname{erf} \sqrt{\left(K_{r}-a_{3}\right) t}\right] \\
& +\left(-a_{4}+a_{6}\right)\left[\sqrt{\frac{\operatorname{Pr}}{\pi t}} \exp \left(-\frac{R t}{\operatorname{Pr}}\right)+\sqrt{R} \operatorname{erf} \sqrt{\frac{R}{\operatorname{Pr}} t}\right] \\
& +a_{5}\left[\sqrt{\frac{\operatorname{Pr}}{\pi t}} \exp \left(-\frac{R t}{\operatorname{Pr}}\right)+\sqrt{R} \operatorname{erf} \sqrt{\frac{R t}{\operatorname{Pr}}}+\frac{\operatorname{Pr}}{2 \sqrt{R}} \operatorname{erf}\left(\sqrt{\frac{R t}{\operatorname{Pr}}}\right)\right] \\
& +\left(a_{4}-a_{6}\right) \exp \left(-a_{3} t\right)\left[\sqrt{\frac{\operatorname{Pr}}{\pi t}} \exp \left(-\frac{R t}{\operatorname{Pr}}\right)+\sqrt{R-a_{3} \operatorname{Pr}} \operatorname{erf} \sqrt{\left(\frac{R}{\operatorname{Pr}}-a_{3}\right) t}\right]
\end{aligned}
$$

\section{Results and discussion}

Equations 12, 13 and 15 with the boundary condition (14) were solved analytically by using integral transform technique. The velocity profiles are represented from Figs. 2 , $3,4,5,6,7,8,9$ and 10 when $R=1, S c=0.22, k=0.5, t=0.4$, $S o=6, G r=4, G m=5, m=1.5, K^{2}=4$ and $\operatorname{Pr}=0.71$. The effects of $M, m, K^{2}, G r, G m$, So and Sc on the velocity field are shown in Figs. 2, 3, 4, 5, 6, 7, 8, 9 and 10 in the presence of radiation, chemical reaction and mass diffusion on the flow field in the boundary layer region. Numerical values of primary $(u)$ and secondary $(w)$ fluid transverse velocities are computed and illustrated through the graphs. Figure 2 depicts the variation of transverse velocity various magnetic parameter values. It is observed that an increase in magnetic parameter $M$ the velocity decreases. It is viewed the fact that the application of transverse magnetic field

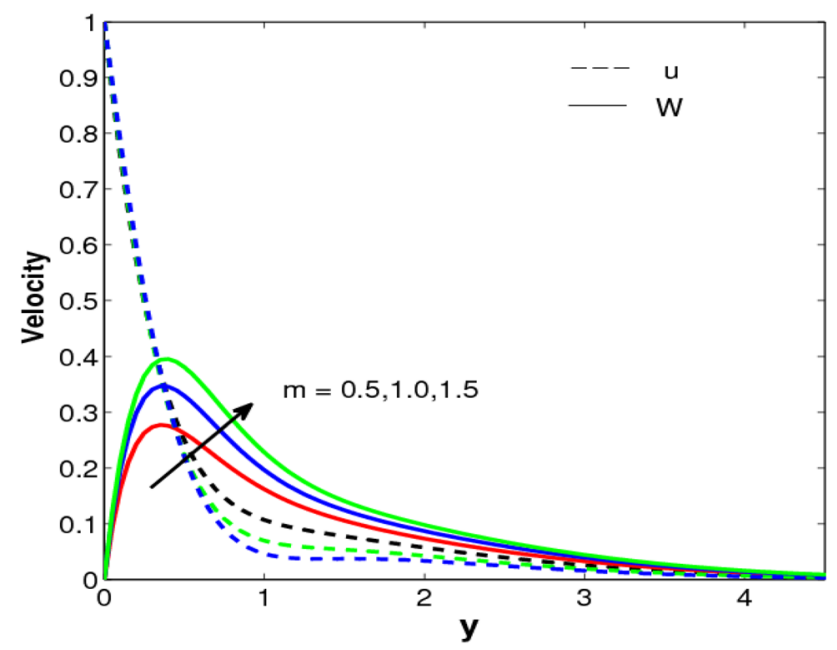

Fig. 3 Transverse velocity profiles for different $m$ 


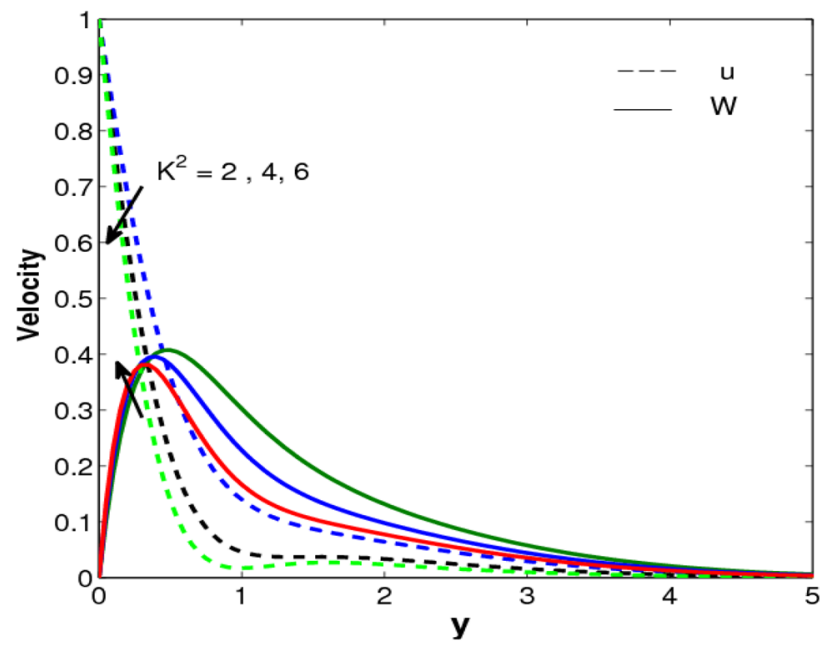

Fig. 4 Transverse velocity profiles for different $K^{2}$

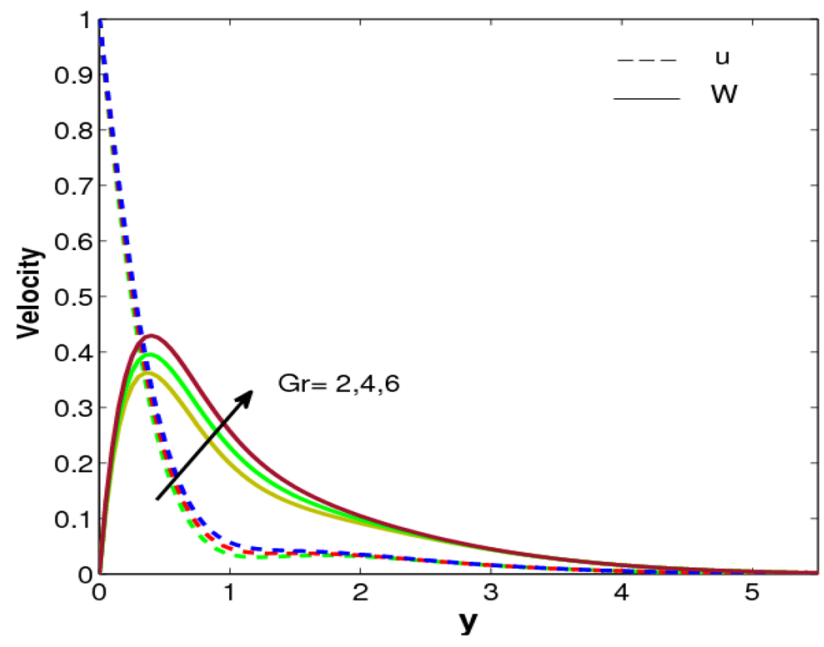

Fig. 5 Transverse velocity profiles for different $G r$

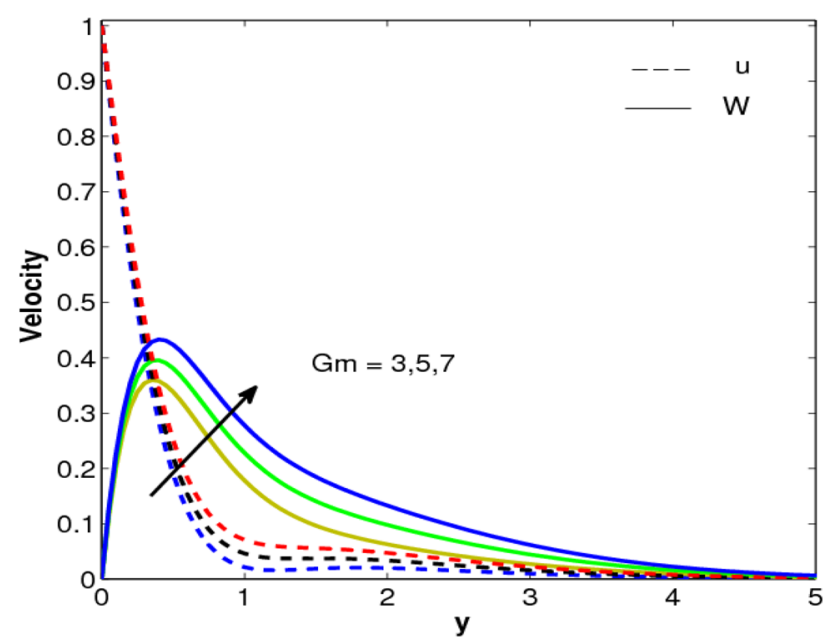

Fig. 6 Transverse velocity profiles for different $G m$

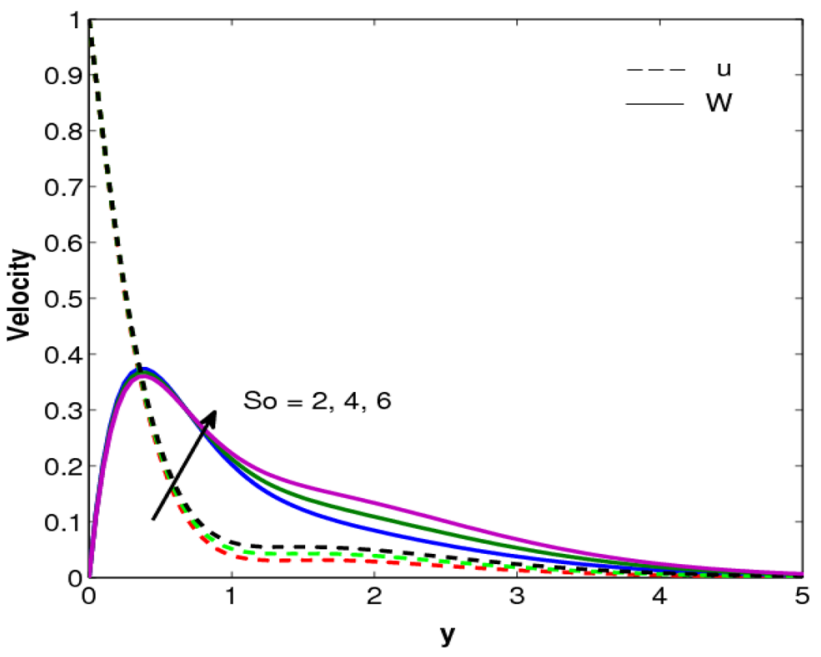

Fig. 7 Transverse velocity profiles for different of So

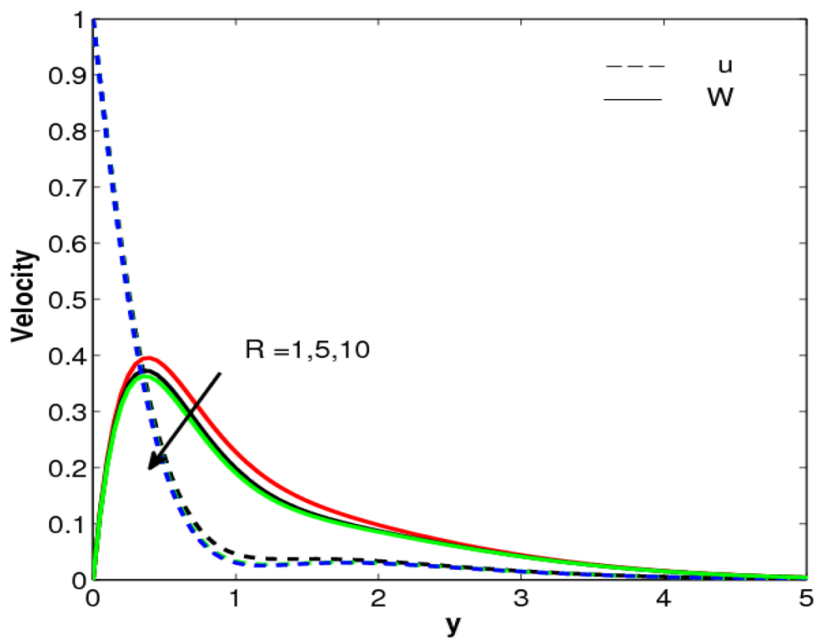

Fig. 8 Transverse velocity profiles for different $R$

will result in a Lorentz force similar to drag force, which tends to resist the fluid flow and thus reducing its velocity in both primary and secondary profiles. Figure 3 reveals the effect of Hall current on velocity field and it is observed that the fluid particles are thermally activated on the application of hall current. Consequently, the generated thermal force accelerates the fluid particles. Therefore, it tends to increase the velocity of fluid particles in both primary and secondary flow directions. Figure 4 shows the effect of rotation parameter on primary and secondary fluid velocities. Primary fluid velocity decreases with the increase of rotation. This is characteristic due to an increase of frictional force. But on the other hand, the secondary velocity is increased as the frictional force is decreased. As a result, 


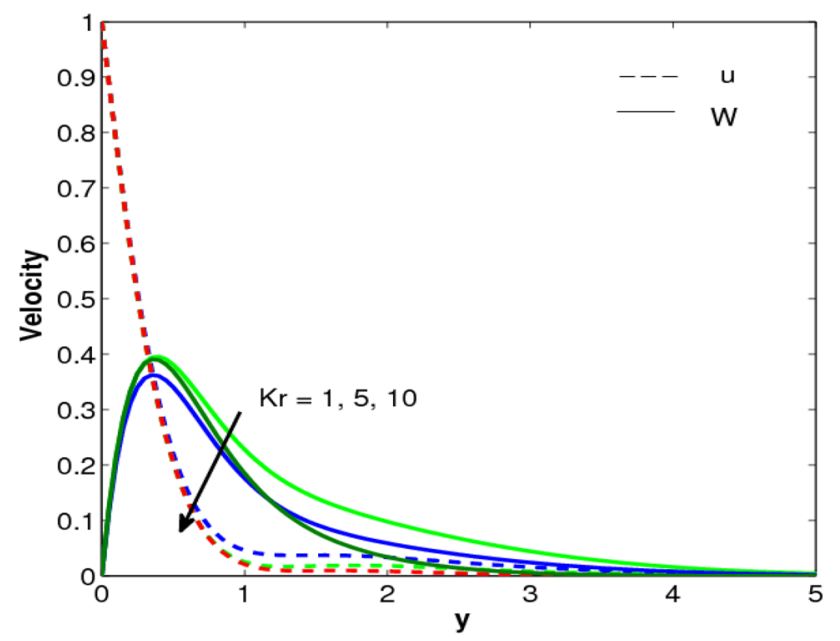

Fig. 9 Transverse velocity profiles for different $K r$

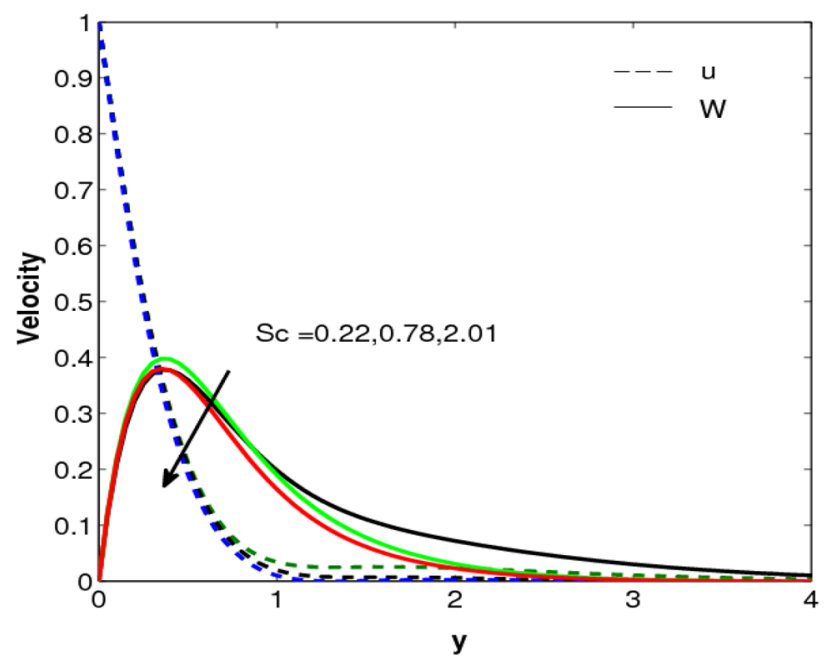

Fig. 10 Transverse velocity profiles for different SC

the fluid particles can set into motion increasing the velocity with rotation.

From Figs. 5, 6 and 7 depicts that the velocity curves reduces with increasing of various parameters $\mathrm{Gr}, \mathrm{Gm}$ and So. This implies that the generated thermal buoyancy and concentration buoyancy force leads to accelerating the fluid particles on both fluid transport flow direction. It is noticed that from Fig. 8 both primary and secondary fluid flow velocity increases with increasing of various parameters $R$. It is fact that the conduction effect of the viscous fluids increment in the presence of thermal radiation. Consequently, higher estimations of $R$ infer higher surface heat flux thus enlarge the temperature inside the boundary layer vicinity. In Fig. 9 reveals that the primary and secondary fluid velocities reduces with increasing magnitude of the chemical reaction parameter, Likewise, we see that

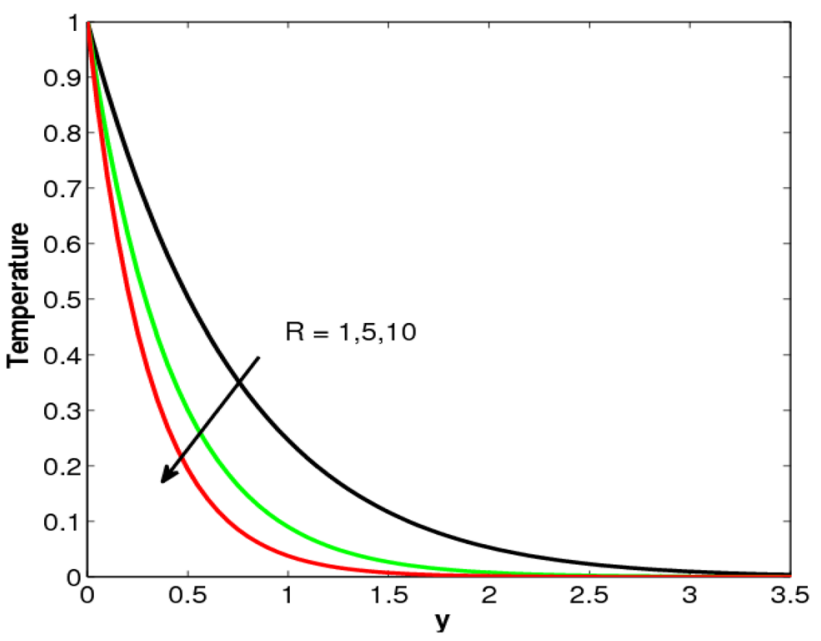

Fig. 11 Temperature profiles for different $R$

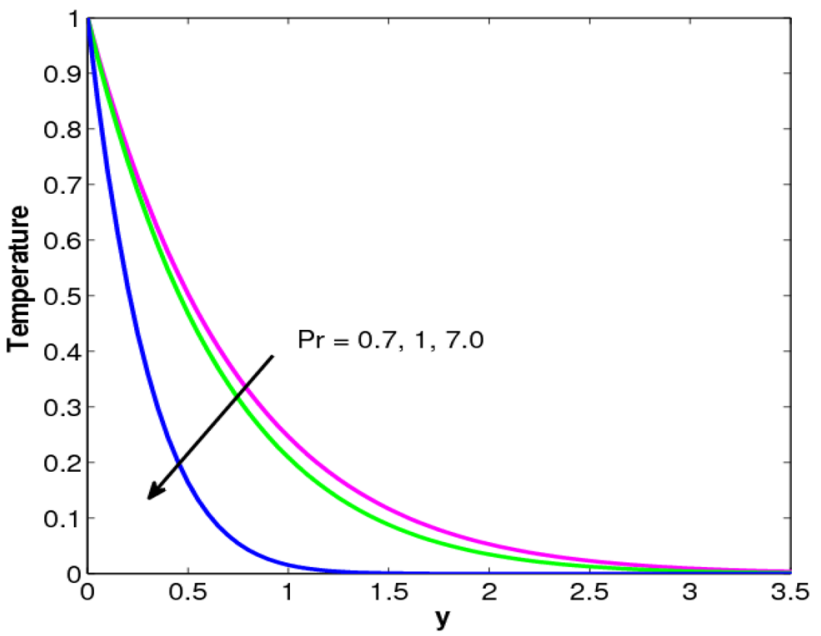

Fig. 12 Temperature profiles for different $\mathrm{Pr}$

the magnitude of the stream wise velocity reduces and the enunciation point in the velocity distribution moves further away from the surface. Figure 10 shows that the transverse velocity profiles reduce with enhancing values of Schmidt number.

The influence of various flow parameters on the fluid temperature is shown in Figs. 11 and 12. From Fig. 11 reveals that with increasing values of radiation parameter the fluid temperature decreases rapidly, hence using radiation we can control the temperature distribution. The Prandtl number is more prominent in categorize the fluids due to its importance in vicinity of the frictionless regime. This number varies the temperature distribution is shown through the Fig. 12, and it is pointed out that the low Prandtl number causes a rise in temperature. The fact is explained, it is because of that low thermal conductivities 
are supposed to get a reduction in conduction; hence at lower values of $\operatorname{Pr}$ contains more spacing in molecular structure within the fluid, thus temperature reduces, agrees with Kim [15].

Concentration profiles for various values of Soret effect So, Schmidt number Sc, Prandtl number Pr, chemical reaction $k$ and time $t$ are shown in Figs. 13, 14, 15, 16 and 17. From Fig. 13 it is seen that the concentration due to variation in Schmidt number for gases like hydrogen $(S c=0.22)$, water-vapor $(S c=0.60)$ and oxygen $(S c=0.78)$ the concentration field steadily for hydrogen and accrues for oxygen in comparison to water-vapor. An increasing in Schmidt number leads to decreases in the concentration boundary layer thickness, agrees with Makinde [19]. Figure 14 reveals that the species concentration decreases with the increasing values of the Prandtl number. In Fig. 15 it is observed that concentration decrease with increasing values of chemical reaction parameter $k$. From Figs. 16 and 17 it is

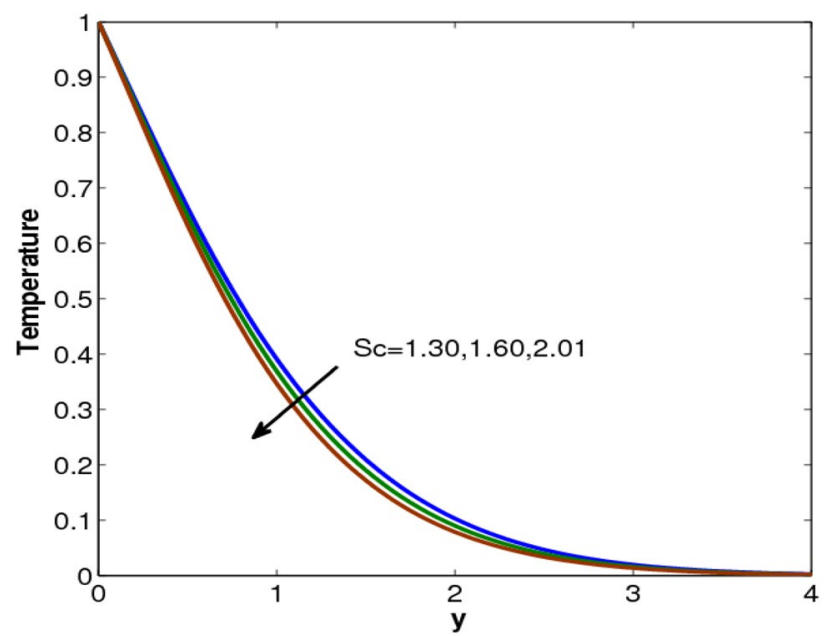

Fig. 13 Concentration profiles for different SC

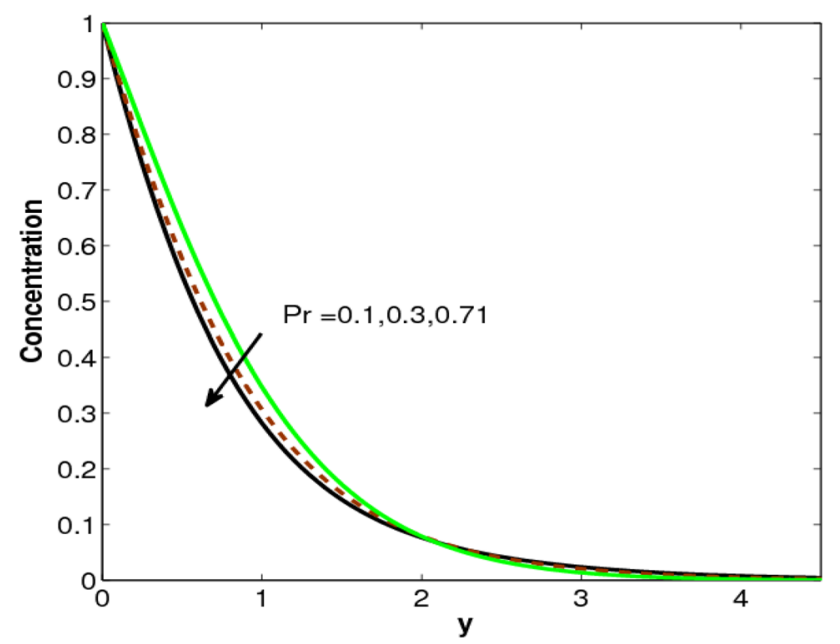

Fig. 14 Concentration profiles for different $\mathrm{Pr}$

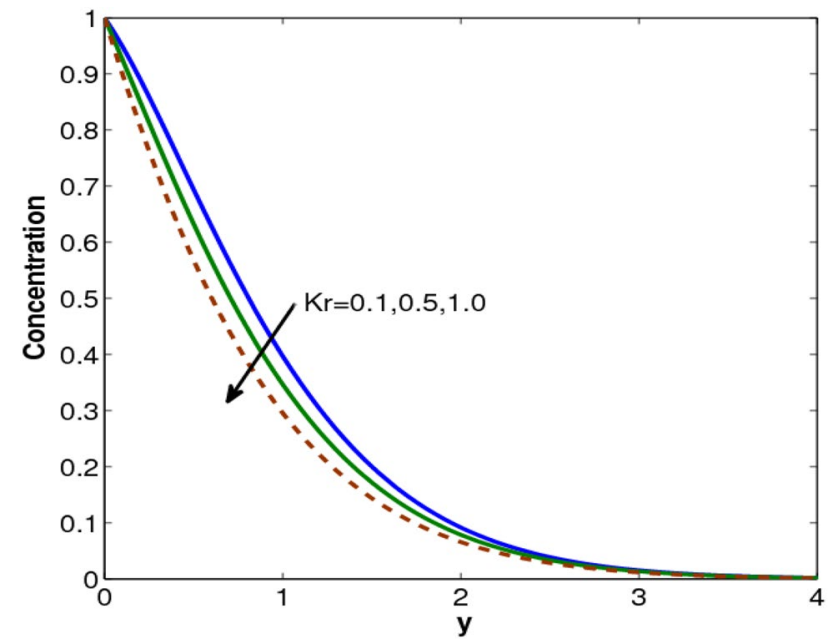

Fig. 15 Concentration profiles for different $\mathrm{Kr}$

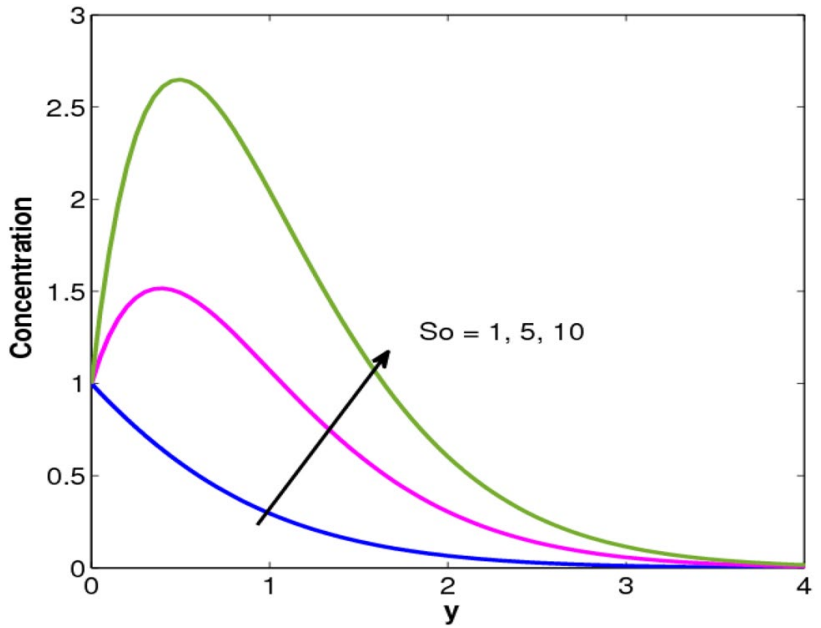

Fig. 16 Concentration profiles for different So

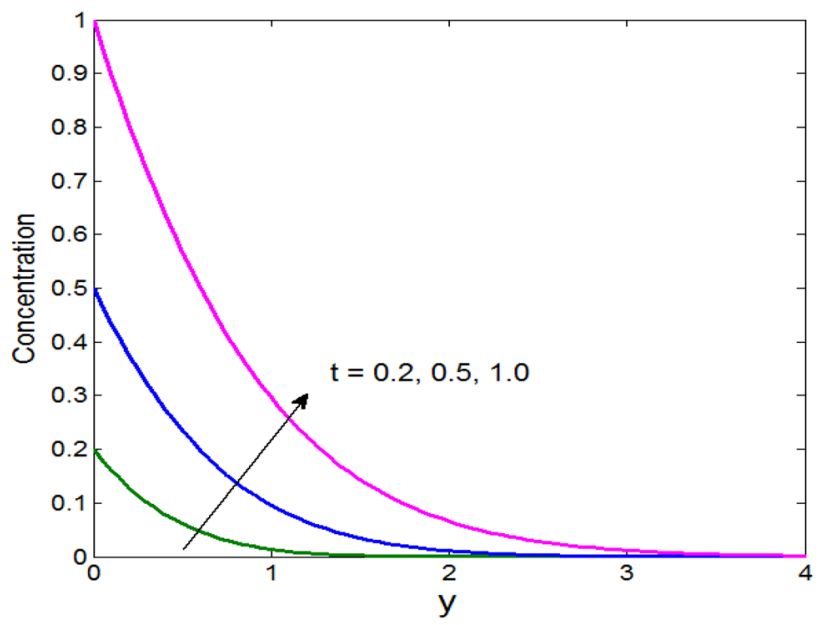

Fig. 17 Concentration profiles for different $t$ 
noticed that the species concentration increases with an increase of $S o$ as well as it increases also with time $t$. Is also noticed that the effects are much stronger near the surface of the solid wall. This indicates that the fluid concentration rises due to the increasing level of thermal diffusion and as a result, species concentration increases.

The skin-friction at the solid wall is presented in the Figs. 18 and 19. From Fig. 18 depicts that the skin-friction at the plate decreases with increasing magnetic parameter. From this figure, it can be understood that the skinfriction near the wall falls with the rise of the magnetic parameter. It is attributed due to an increase of restricting force in both primary and secondary. From Fig. 19 illustrate the skin-friction at the solid wall increases with shrinking values So. It is noticed that the effects are much stronger near the surface of the solid boundary.

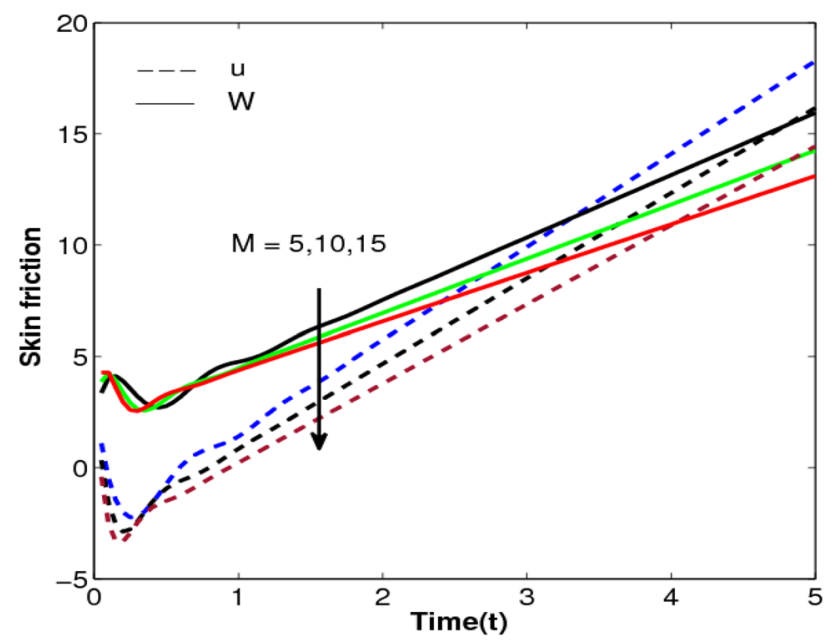

Fig. 18 Skin-friction for various values of $M$

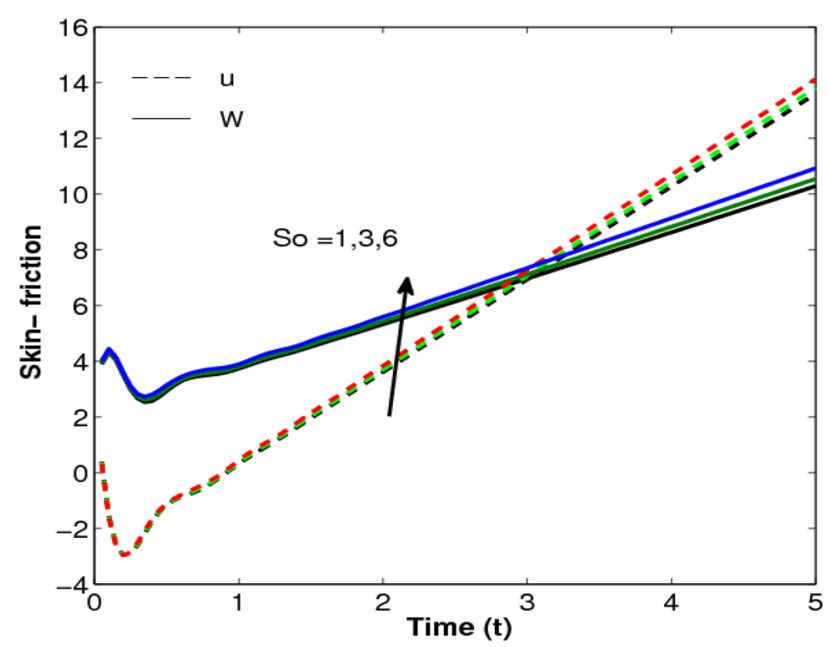

Fig. 19 Skin-friction for various values of So
From Fig. 20 reveals that the Nusselt number presented against time $t$. The Nusselt number increases with an increase in radiation parameter $R$ or the increasing values of Prandtl number. And it is also observed that the rate at which heat transfer changes water is more than the conducting air. It may happen caused by that slighter values of Pr are comparable same to increasing the ability of material strength and thus energy transition is capable to scatter far enough from the wall more speedily rather than at the superior values of $\operatorname{Pr}$, consequently, the gradient temperature is diminished. Figure 21 illustrate the Sherwood number tendency reporting time $t$ verses it. It is remarkably noted that the species diffusion rate very closed to the solid wall, decreases as So rises in magnitude. This is attributed due to the concentration boundary thickness is high and as a result, the rate of mass transfer reduces.

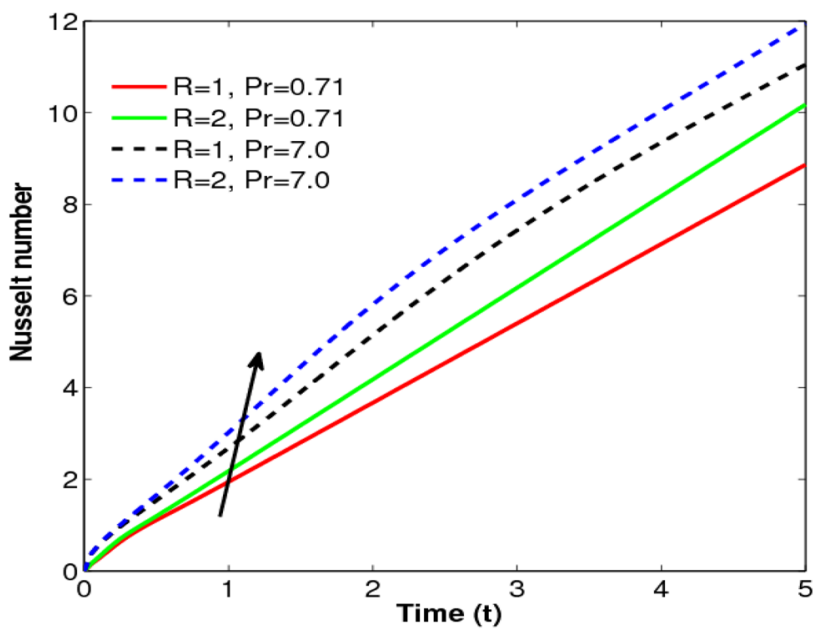

Fig. 20 Nusselt number for various values of $\operatorname{Pr}$ and $R$

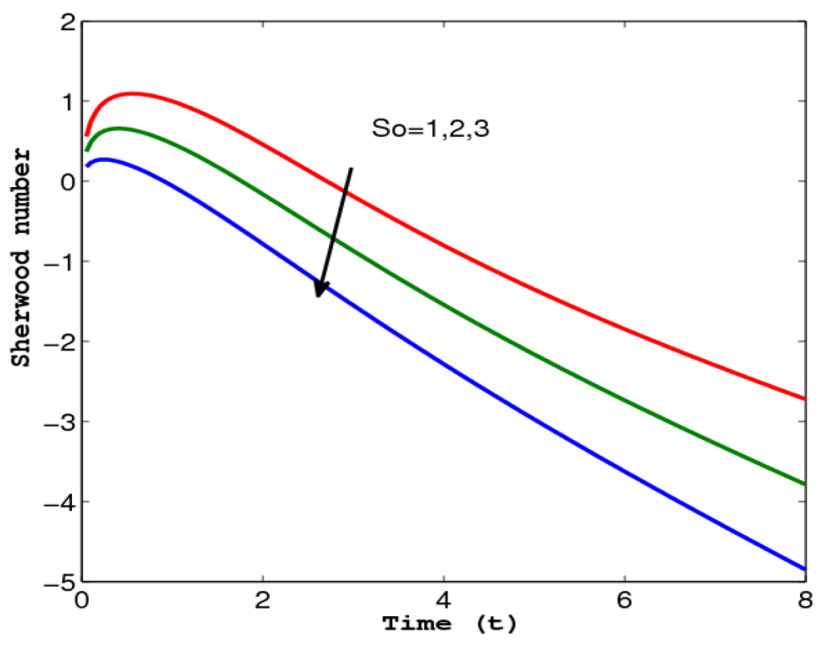

Fig. 21 Sherwood number for various values of So 


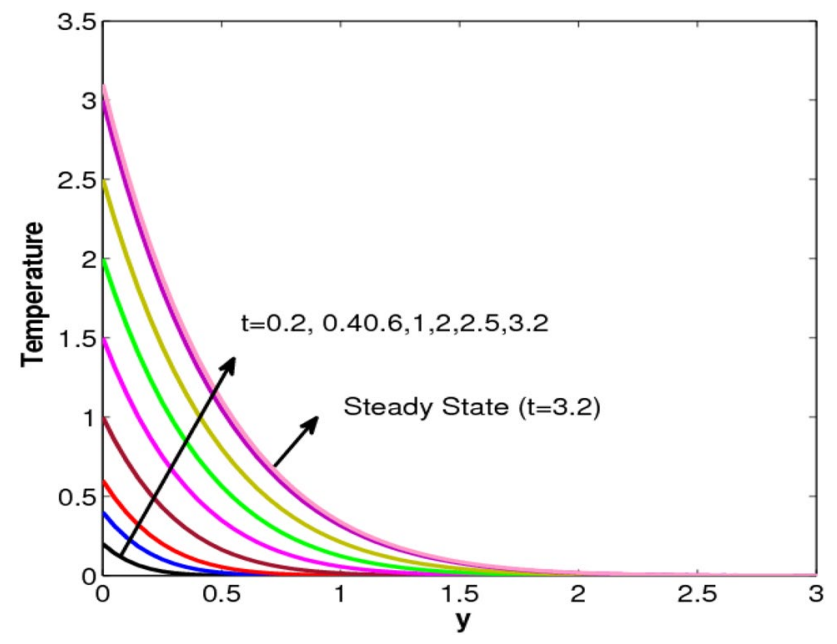

Fig. 22 Sherwood number for various values of $t$

In Fig. 22 shows that the temperature profile is increasing with time until it achieves steady state conditions. This is due to the heat transferred from the plate to the fluid increases with time which in turn raises the fluid temperature. The temperature decreases gradually along $y$ until it satisfies the free stream condition.

\section{Conclusions}

Analytical solutions have been derived for the effects of thermal diffusion and hall current, radiation on unsteady MHD free convective viscous, incompressible, electrically conducting chemically reacting fluid past an impulsively moving infinite vertical plate embedded in a fluid saturated porous medium with variable temperature and variable concentration under the influence of uniform transverse magnetic field. The expressions for the velocity, the temperature and concentration have been obtained in closed form with the help of integral transform technique. The effects of the appropriate parameters on velocity, temperature and concentration profiles are presented graphically. The conclusions of the study are as follows:

- The temperature in degrees of hotness becomes smaller when the radiation parameter tends to rise.

- The amount of energy within the structure of the molecule reduces with upgrading Prandtl number.

- The species of concentration escalates with deceleration of Soret number.

- The fluid transport becomes faster when the magnetic parameter shrinks.

- The fluid velocity enlarges with shrinking values of Soret number.

- The Nusselt number getting an improvement when Prandtl number or radiation become strengthen.

- Sherwood number decelerates when Soret number intensify.

- Skin-friction tends to become slower by strengthen magnetic parameter.

\section{Compliance with ethical standards}

Conflict of interest The author declare that they have no competing interests. 


\section{Appendix}

$$
\begin{aligned}
& B_{1}=\left[\begin{array}{c}
\left(\frac{t}{2}+\frac{y \sqrt{S c}}{4 \sqrt{K_{r}}}\right) \exp \left(y \sqrt{K_{r} S c}\right) \operatorname{erfc}\left(\frac{y \sqrt{s c}}{2 \sqrt{t}}+\sqrt{K_{r} t}\right) \\
+\left(\frac{t}{2}-\frac{y \sqrt{S c}}{4 \sqrt{K_{r}}}\right) \exp \left(-y \sqrt{K_{r} S c}\right) \operatorname{erfc}\left(\frac{y \sqrt{S c}}{2 \sqrt{t}}-\sqrt{K_{r} t}\right)
\end{array}\right] \\
& B_{2}=\left[\exp \left(y \sqrt{K_{r} S c}\right) \operatorname{erfc}\left(\frac{y \sqrt{S c}}{2 \sqrt{t}}+\sqrt{K_{r} t}\right)+\exp \left(-y \sqrt{K_{r} S c}\right) \operatorname{erfc}\left(\frac{y \sqrt{S c}}{2 \sqrt{t}}-\sqrt{K_{r} t}\right)\right] \\
& B_{3}=\left[\begin{array}{c}
\exp \left(y \sqrt{\left(K_{r}-a_{3}\right) S c}\right) \operatorname{erfc}\left(\frac{y \sqrt{s c}}{2 \sqrt{t}}+\sqrt{\left(K_{r}-a_{3}\right) t}\right) \\
+\exp \left(-y \sqrt{\left(K_{r}-a_{3}\right) S c}\right) \operatorname{erfc}\left(\frac{y \sqrt{s c}}{2 \sqrt{t}}-\sqrt{\left(K_{r}-a_{3}\right) t}\right)
\end{array}\right] \\
& B_{4}=\left[\exp (y \sqrt{R}) \operatorname{erfc}\left(\frac{y \sqrt{\operatorname{Pr}}}{2 \sqrt{t}}+\sqrt{\frac{R t}{\operatorname{Pr}}}\right)+\exp (-y \sqrt{R}) \operatorname{erfc}\left(\frac{y \sqrt{\operatorname{Pr}}}{2 \sqrt{t}}-\sqrt{\frac{R t}{\operatorname{Pr}}}\right)\right] \\
& B_{5}=\left[\left(\frac{t}{2}+\frac{y \operatorname{Pr}}{4 \sqrt{R}}\right) \exp (y \sqrt{R}) \operatorname{erfc}\left(\frac{y \sqrt{\operatorname{Pr}}}{2 \sqrt{t}}+\sqrt{\frac{R t}{\operatorname{Pr}}}\right)+\left(\frac{t}{2}-\frac{y \operatorname{Pr}}{4 \sqrt{R}}\right) \exp (y-\sqrt{R}) \operatorname{erfc}\left(\frac{y \sqrt{\operatorname{Pr}}}{2 \sqrt{t}}-\sqrt{\frac{R t}{\operatorname{Pr}}}\right)\right] \\
& B_{6}=\left[\exp \left(y \sqrt{R-a_{3} \operatorname{Pr}}\right) \operatorname{erfc}\left(\frac{y \sqrt{\operatorname{Pr}}}{2 \sqrt{t}}+\sqrt{\frac{R t}{\operatorname{Pr}}-a_{3} t}\right)+\exp \left(-y \sqrt{R-a_{3} \operatorname{Pr}}\right) \operatorname{erfc}\left(\frac{y \sqrt{\operatorname{Pr}}}{2 \sqrt{t}}-\sqrt{\frac{R t}{\operatorname{Pr}}-a_{3} t}\right)\right] \\
& B_{7}=\left[\exp (y \sqrt{b}) \operatorname{erfc}\left(\frac{y}{2 \sqrt{t}}+\sqrt{b t}\right)+\exp (-y \sqrt{b}) \operatorname{erfc}\left(\frac{y}{2 \sqrt{t}}-\sqrt{b t}\right)\right] \\
& B_{8}=\left[\left(\frac{t}{2}+\frac{y}{4 \sqrt{b}}\right) \exp (y \sqrt{b}) \operatorname{erfc}\left(\frac{y}{2 \sqrt{t}}+\sqrt{b t}\right)+\left(\frac{t}{2}-\frac{y}{4 \sqrt{b}}\right) \exp (-y \sqrt{b}) \operatorname{erfc}\left(\frac{y}{2 \sqrt{t}}-\sqrt{b t}\right)\right] \\
& B_{9}=\left[\exp \left(y \sqrt{b-a_{8}}\right) \operatorname{erfc}\left(\frac{y}{2 \sqrt{t}}+\sqrt{\left(b-a_{8}\right) t}\right)+\exp \left(-y \sqrt{b-a_{8}}\right) \operatorname{erfc}\left(\frac{y}{2 \sqrt{t}}-\sqrt{\left(b-a_{8}\right) t}\right)\right] \\
& B_{10}=\left[\exp \left(y \sqrt{b-a_{8}}\right) \operatorname{erfc}\left(\frac{y}{2 \sqrt{t}}+\sqrt{\left(b-a_{8}\right) t}\right)+\exp \left(-y \sqrt{b-a_{8}}\right) \operatorname{erfc}\left(\frac{y}{2 \sqrt{t}}-\sqrt{\left(b-a_{8}\right) t}\right)\right]
\end{aligned}
$$




$$
\begin{aligned}
& B_{11}=\left[\exp \left(y \sqrt{b-a_{3}}\right) \operatorname{erfc}\left(\frac{y}{2 \sqrt{t}}+\sqrt{\left(b-a_{3}\right) t}\right)+\exp \left(-y \sqrt{b-a_{3}}\right) \operatorname{erfc}\left(\frac{y}{2 \sqrt{t}}-\sqrt{\left(b-a_{3}\right) t}\right)\right] \\
& B_{12}=\left[\exp (y \sqrt{R}) \operatorname{erfc}\left(\frac{y \sqrt{\operatorname{Pr}}}{2 \sqrt{t}}+\sqrt{\frac{R t}{\operatorname{Pr}}}\right)+\exp (-y \sqrt{R}) \operatorname{erfc}\left(\frac{y \sqrt{\operatorname{Pr}}}{2 \sqrt{t}}-\sqrt{\frac{R t}{\operatorname{Pr}}}\right)\right] \\
& B_{13}=\left[\left(\frac{t}{2}+\frac{y \operatorname{Pr}}{4 \sqrt{R}}\right) \exp (y \sqrt{R}) \operatorname{erfc}\left(\frac{y \sqrt{\operatorname{Pr}}}{2 \sqrt{t}}+\sqrt{\frac{R t}{\operatorname{Pr}}}\right)+\left(\frac{t}{2}-\frac{y \operatorname{Pr}}{4 \sqrt{R}}\right) \exp (y-\sqrt{R}) \operatorname{erfc}\left(\frac{y \sqrt{\operatorname{Pr}}}{2 \sqrt{t}}-\sqrt{\frac{R t}{\operatorname{Pr}}}\right)\right] \\
& B_{14}=\left[\exp \left(y \sqrt{R-a_{8} \operatorname{Pr}}\right) \operatorname{erfc}\left(\frac{y \sqrt{\operatorname{Pr}}}{2 \sqrt{t}}+\sqrt{\frac{R t}{\operatorname{Pr}}-a_{8} t}\right)+\exp \left(-y \sqrt{R-a_{8} \operatorname{Pr}}\right) \operatorname{erfc}\left(\frac{y \sqrt{\operatorname{Pr}}}{2 \sqrt{t}}-\sqrt{\frac{R t}{\operatorname{Pr}}}-a_{8} t\right)\right] \\
& B_{15}=\left[\exp \left(y \sqrt{R-a_{10} \operatorname{Pr}}\right) \operatorname{erfc}\left(\frac{y \sqrt{\operatorname{Pr}}}{2 \sqrt{t}}+\sqrt{\frac{R t}{\operatorname{Pr}}-a_{10} t}\right)+\exp \left(-y \sqrt{R-a_{10} \operatorname{Pr}}\right) \operatorname{erfc}\left(\frac{y \sqrt{\operatorname{Pr}}}{2 \sqrt{t}}-\sqrt{\frac{R t}{\operatorname{Pr}}-a_{10} t}\right)\right] \\
& B_{16}=\left[\exp \left(y \sqrt{R-a_{3} \operatorname{Pr}}\right) \operatorname{erfc}\left(\frac{y \sqrt{\operatorname{Pr}}}{2 \sqrt{t}}+\sqrt{\frac{R t}{\operatorname{Pr}}-a_{3} t}\right)+\exp \left(-y \sqrt{R-a_{3} \operatorname{Pr}}\right) \operatorname{erfc}\left(\frac{y \sqrt{\operatorname{Pr}}}{2 \sqrt{t}}-\sqrt{\frac{R t}{\operatorname{Pr}}-a_{3} t}\right)\right] \\
& B_{17}=\left[\exp \left(y \sqrt{K_{r} S c}\right) \operatorname{erfc}\left(\frac{y \sqrt{S c}}{2 \sqrt{t}}+\sqrt{K_{r} t}\right)+\exp \left(-y \sqrt{K_{r} S c}\right) \operatorname{erfc}\left(\frac{y \sqrt{S c}}{2 \sqrt{t}}-\sqrt{K_{r} t}\right)\right] \\
& B_{18}=\left[\begin{array}{c}
\left(\frac{t}{2}+\frac{y \sqrt{S c}}{4 \sqrt{K_{r}}}\right) \exp \left(y \sqrt{K_{r} S c}\right) \operatorname{erfc}\left(\frac{y \sqrt{S c}}{2 \sqrt{t}}+\sqrt{K_{r} t}\right) \\
+\left(\frac{t}{2}-\frac{y \sqrt{S c}}{4 \sqrt{K_{r}}}\right) \exp \left(-y \sqrt{K_{r} S c}\right) \operatorname{erfc}\left(\frac{y \sqrt{S c}}{2 \sqrt{t}}-\sqrt{K_{r} t}\right)
\end{array}\right] \\
& B_{19}=\left[\begin{array}{l}
\exp \left(y \sqrt{\left(K_{r}-a_{10}\right) S c}\right) \operatorname{erfc}\left(\frac{y \sqrt{s c}}{2 \sqrt{t}}+\sqrt{\left(K_{r}-a_{10}\right) t}\right) \\
\quad+\exp \left(-y \sqrt{\left(K_{r}-a_{10}\right) S c}\right) \operatorname{erfc}\left(\frac{y \sqrt{S c}}{2 \sqrt{t}}-\sqrt{\left(K_{r}-a_{10}\right) t}\right)
\end{array}\right] \\
& B_{20}=\left[\begin{array}{c}
\exp \left(y \sqrt{\left(K_{r}-a_{3}\right) S c}\right) \operatorname{erfc}\left(\frac{y \sqrt{S c}}{2 \sqrt{t}}+\sqrt{\left(K_{r}-a_{3}\right) t}\right) \\
\quad+\exp \left(-y \sqrt{\left(K_{r}-a_{3}\right) S c}\right) \operatorname{erfc}\left(\frac{y \sqrt{S c}}{2 \sqrt{t}}-\sqrt{\left(K_{r}-a_{3}\right) t}\right)
\end{array}\right]
\end{aligned}
$$

\section{References}

1. Cramer KR, Pai SI (1973) Magneto fluid dynamics for engineers and applied physicists. McGraw-Hill Book Co, New York

2. Cheng P, Minkowycz J (1977) Free convection about a vertical flat plate embedded in a porous medium with application to heat transfer from a dike. J Geophys Res 82(14):2040-2044. https ://doi.org/10.1029/jb082i014p02040

3. Nakayama A, Koyama H (1987) A general similarity transformation for combined free and forced-convection flows within a fluid-saturated porous medium. J Heat Trans 9(4):1041-1045. https://doi.org/10.1115/1.3248180

4. Lai FC, Kulacki FA (1991) Non-darcy mixed convection along a vertical wall in a saturated porous medium. J Heat Trans 113(1):252. https://doi.org/10.1115/1.2910537

5. Hsieh JC, Chen TS, Armaly BF (1993) Non-similarity solutions for mixed convection from vertical Surfaces in porous medium variable surface temperature or heat flux. Int J Heat Mass Trans 36(6):1485-1493. https://doi.org/10.1016/s0017-9310(05)80059 $-6$

6. Nield DA, Kuznetsov AV (2009) The Cheng-Minkowycz problem for natural convective boundary-layer flow in a porous medium saturated by a nanofluid. Int J Heat Mass Trans 52(25):57925795. https://doi.org/10.1016/j.ijheatmasstransfer.2009.07.024

7. Gorla RSR, Chamkha A (2011) Natural convective boundary layer flow over a non-isothermal vertical plate embedded in a porous medium saturated with a nanofluid. Nanosc Microsc Thermophys Eng 15(2):81-94. https://doi.org/10.1080/15567 265.2010.549931

8. Nield DA, Bejan A (2006) Convection in porous media, 3rd edn. Springer, New York

9. Vafai K (2005) Handbook of porous media, 2nd edn. Taylor and Francis Group, Florida. https://doi.org/10.1201/9780415876384

10. Pop I, Ingham DB (2002) Transport phenomena in porous mediaII, 1st edn. Elsevier, Oxford. https://doi.org/10.1016/b978-00804 3965-5/50000-3

11. Raptis A, Kafousias N (1982) Heat transfer in flow through a porous medium bounded by an infinite vertical plate under the action of a magnetic field. Int J Energy Res 6(3):241-245. https ://doi.org/10.1002/er.444006030

12. Raptis AA (1986) Flow through a porous medium in the presence of a magnetic field. Int J Energy Res 10(1):97-100. https://doi. org/10.1002/er.4440100112

13. Chamkha AJ (1997) Hydromagnetic natural convection from an isothermal inclined surface adjacent to a thermally stratified 
porous medium. Int J Eng Sci 35(10-11):975-986. https://doi. org/10.1016/s0020-7225(96)00122-x

14. Aldoss TK, Al-Nimr MA, Jarrah M, Al-Sha'er BJ (1995) Magnetohydrodynamic mixed convection from a vertical plate embedded in a porous medium. Numer Heat Trans Part A Appl 28:635-645. https://doi.org/10.1080/10407789508913766

15. Kim YJ (2000) Unsteady MHD convective heat transfer past a semi-infinite vertical porous moving plate with variable suction. Int J Eng Sci 38:833-845

16. Ibrahim FS, Hassanien IA, Bakr AA (2004) Unsteady magnetohydrodynamic micro polar fluid flow and heat transfer over a vertical porous plate through a porous medium in the presence of thermal and mass diffusion with a constant heat source. Can J Phys 82(10):775-790. https://doi.org/10.1139/p04-021

17. Alam MdS, Rahman MdM (2009) Dufour and Soret effects on MHD free convective heat and mass transfer flow past a vertical porous flat plate embedded in a porous medium. JNAME 2:55-65. https://doi.org/10.3329/jname.v2i1.2030

18. Makinde OD, Ogulu A (2008) The effect of thermal radiation on the heat and mass transfer flow of a variable viscosity fluid past a vertical porous plate permeated by a transverse magnetic field. Chem Eng Commun 195(12):1575-1584. https://doi. org/10.1080/00986440802115549

19. Makinde OD (2009) On MHD boundary-layer flow and mass transfer past a vertical plate in a porous medium with constant heat flux International. J Numer Methods Heat Fluid Flow 19(3/4):546-554. https://doi.org/10.1108/09615530910938434

20. Cess RD (1966) The interaction of thermal radiation with free convection heat transfer. Int J Heat Mass Trans 9:1269-1277

21. Hossain MA, Takhar HS (1996) Radiation effect on mixed convection along a vertical plate with uniform surface temperature. Heat Mass Trans 31(4):243-248. https://doi.org/10.1007/bf023 28616

22. Bakier AY, Gorla RSR (1996) Thermal radiation effect on mixed convection from horizontal surfaces in saturated porous media. Transp Porous Media 23(3):357-363. https://doi.org/10.1007/ bf00167104

23. Chamkha AJ (2000) Thermal radiation and buoyancy effects on hydromagnetic flow over an accelerating permeable surface with heat source or sink. Int J Eng Sci 38(15):1699-1712. https ://doi.org/10.1016/s0020-7225(99)00134-2

24. Israel-Cookey C, Ogulu A, Omubo-Pepple VB (2003) Influence of viscous dissipation and radiation on unsteady MHD free-convection flow past an infinite heated vertical plate in a porous medium with time-dependent suction. Int J Heat Mass Trans 46(13):2305-2311. https://doi.org/10.1016/s0017 $-9310(02) 00544-6$

25. Muthucumaraswamy R (2003) Effects of chemical reaction on moving isothermal vertical plate with variable mass diffusion. Theor Appl Mech 30:209-220. https://doi.org/10.2298/tam03 03209

26. Makinde OD, Sibanda P (2008) Magneto-hydrodynamic mixedconvective flow and heat and mass transfer past a vertical plate in a porous medium with constant wall suction. J Heat Trans 130(11):112602. https://doi.org/10.1115/1.2955471

27. Mostafa A, Mahmoud A (2009) Thermal radiation effect on unsteady MHD free convection flow past a vertical plate with temperature-dependent viscosity. Can J Chem Eng 87(1):47-52. https://doi.org/10.1002/cjce.20135

28. Kumar BR, Kumar TS, Vijaya Kumar AG (2015) Thermal diffusion and radiation Effects on unsteady free convection flow in the presence of magnetic field fixed relative to the fluid or to the plate. JFHMT 6:6-12. https://doi.org/10.5098/hmt.6.12
29. Nandkeolyar R, Das M, Sibanda P (2013) Unsteady hydromagnetic heat and mass transfer flow of a heat radiating and chemically reactive fluid past a flat porous plate with ramped wall temperature. Math Probl Eng 2013:1-12. https://doi. org/10.1155/2013/381806

30. Seth GS, Ansari MdS, Nandkeolyar R (2010) MHD natural convection flow with radiative heat transfer past an impulsively moving plate with ramped wall temperature. Heat Mass Trans 47(5):551-561. https://doi.org/10.1007/s00231-010-0740-1

31. Das S, Sarkar BC, Jana RN (2012) Hall effect on MHD free convection boundary layer flow past a vertical flat plate. Meccanica 48(6):1387-1398. https://doi.org/10.1007/s11012-012-9673-8

32. Seth GS, Sarkar S, Hussain SM (2014) Effects of hall current, radiation and rotation on natural convection heat and mass transfer flow past a moving vertical plate. Ain Shams Eng J 5(2):489-503. https://doi.org/10.1016/j.asej.2013.09.014

33. Seth GS, Hussain SM, Sarkar S (2014) Effects of hall current and rotation on unsteady MHD natural convection flow with heat and mass transfer past an impulsively moving vertical plate in the presence of radiation and chemical reaction. Bulg Chem Commun 46(4):704-718

34. Takha HS, Gorla RSR, Soundalgekar VM (1996) Short communication radiation effects on MHD free convection flow of a gas past a semi-infinite vertical plate. Int Methods for Heat Fluid Flow 6(2):77-83. https://doi.org/10.1108/09615539610113118

35. Mythreye A, Pramod JP, Balamurugan KS (2015) Chemical reaction on unsteady MHD convective heat and mass transfer past a semi-infinite vertical permeable moving plate with heat absorption. Procedia Eng 127:613-620. https://doi.org/10.1016/j.proen g.2015.11.352

36. Rashad AM, Rashidi MM, Lorenzini G, Ahmed SE, Aly AM (2017) Magnetic field and internal heat generation effects on the free convection in a rectangular cavity filled with a porous medium saturated with $\mathrm{Cu}$-water nanofluid. Int J Heat Mass Trans 104:878-889. https://doi.org/10.1016/j.ijheatmasstrans fer.2016.08.025

37. Bhatti MM, Mishra SR, Abbas T, Rashidi MM (2018) A mathematical model of MHD nanofluid flow having gyrotactic microorganisms with thermal radiation and chemical reaction effects. Neural Comput Appl 30(4):1237-1249. https://doi.org/10.1007/ s00521-016-2768-8

38. Yuan M, Rasul M, Rashidi MM, Yang Z (2019) MHD convective heat transfer of $\mathrm{Ag}-\mathrm{MgO} /$ water hybrid nanofluid in a channel with active heaters and coolers. Int J Heat Mass Trans 137:714726. https://doi.org/10.1016/j.ijheatmasstransfer.2019.03.169

39. Yuan M, Rasul M, Rashidi MM, Manca O, Yang Z (2019) Numerical investigation of MHD effects on nanofluid heat transfer in a baffled U-shaped enclosure using lattice Boltzmann method. J Therm Anal Calorim 135(6):3197-3213. https://doi.org/10.1007/ s10973-018-7518-y

40. Yuan M, Rasul M, Rashidi MM, Yang Z, Mikhail AS (2019) Numerical study of MHD nanofluid natural convection in a baffled U-shaped enclosure. Int J Heat Mass Trans 130:123-134. https ://doi.org/10.1016/j.ijheatmasstransfer.2018.10.072

Publisher's Note Springer Nature remains neutral with regard to jurisdictional claims in published maps and institutional affiliations. 